\title{
Multi-touch GIS Based on Optical and Computer Vision
}

\author{
Bo Wan, Dongxue Zhao, Xiu Wen, Gengdai Liu \\ Computer Science Institute, Xidian University, Xi'an, Shannxi, China
}

\begin{abstract}
This paper describes a multi-touch GIS(Geography Information System) based on optical and computer vision. In the system, user can operates map directly through gesture. A series of image processing and computation of video frames is used to get data and trajectory of touch point according to the characteristics of touch point. Some multi-touch gestures corresponding to typical GIS operation and its state transition model are defined. According to these definitions, a gesture recognition method based on geometric characteristics of trajectory is given. This method can recognize the trajectory into correct interaction gestures and operate the GIS through MapX.
\end{abstract}

Keywords: GIS ; multi-touch ; gesture recognition

\section{Introduction}

In the multi-touch technology, multitouch technology based on optical and computer vision has more advantages in response speed and touch accuracy than other technology based on the capacitive, resistive and surface acoustic wave. In [1], five technologies are given by NUI Group(Natural User Interface Group) which can build a stable multi-touch platform based on optical and computer vision: FTIR(Frustrated Total Internal Reflection), Front and Rear-DI(Front and Rear Diffused Illumination), LLP(Laser Light Plane), LED-LP(Light-emitting Di- ode Light Plane), and DSI(Diffuse Surface Illumination). Microsoft developed the "Microsoft Surface" using the RearDI with a number of projectors, digital cameras and infrared sensors. In [2], the author introduced a multi-touch sensor which used the FTIR technique applicable to large interactive display device, such as interactive wall and interactive desktop. Yue Shi et al [3] developed uTable platform which also used infrared light, cameras and projector. On this basis they also developed JuTable suitable for the multiplayer file sharing system. The touch command seminar system discussed in [4] is build by infrared light and camera also, and applied to military geographic information system. Sangbong et al [5] implemented a FTIR Multi-touch table that consists of sheet of acrylic, infrared LEDs, camera and rear projector, and design new gestures on the Multitouch table. In [6] the authors present a slim tabletop interface with a large HD LED display. Its hybrid recognition method also combines FTIR and LLP.

In current, multi-touch technology based on optical and computer vision has been fully applied in a variety of touch recognition system, but the vast majority of GIS applications still use the mouse and keyboard to complete operation other than work conjunction with multi-touch technology. For example, Xu Chengzhi et al [7] designed New Spatial Query Language Based on GIS in the ArcGIS platform. Wang Dewen et al [8] used MapX controls to built GIS. Song Xueyuan et al [9] developed GPS Position Information 
Real-time mark system based on MapX, etc.

The multi-touch GIS presented in this paper adopts the Rear-DI technology. It consists of computer, infrared camera and projector. Manipulating GIS by fingers directly is allowed, which is more intuitive for the users. A series of image processing and computing of video frames is used to get data and trajectory of touch point according to the characteristics of touch point. Some multi-touch gestures corresponding to typical GIS operation and its state transition model are defined in the GIS module. According to these definitions, a gesture recognition method based on geometric characteristics of trajectory is given. This method can recognize the trajectory into correct interaction gestures and operate the GIS through MapX. The contributions of this paper are as follows:

- A GIS which can be manipulated directly by fingers is developed, which has better human-computer interaction features and user experience contrast to the traditional GIS.

- A simple and efficient touch point tracing algorithm is given in this article, which can accurately trace the touch point and capture touch point trajectory.

- Multi-touch gestures corresponding to GIS operation and its state transition model are defined in the system. According to these definitions, a gesture recognition method based on the geometric characteristic of touch point trajectory is given. This method can recognize the trajectory into correct interaction gestures.

\section{Multi-touch GIS Overview}

\subsection{The Architecture of multi-touch GIS}

The platform consists of both hardware and software. The hardware part includes: 850nm infrared light diode array, acrylic material touch screen, camera, computer and projector. The infrared light diode array is used to provide infrared light. The touch screen can display the GIS map and accept user touch operation. The camera captures the infrared light image reflected by finger. Computer completes touch point tracing, gesture recognition and GIS operation corresponding to gesture, such as: click, zoom in, and zoom out and so on. The projector is used to display the final results. The software part of our system consists of the GIS module and the touch point tracing module. Figure 1 illustrates the architecture of our GIS platform.

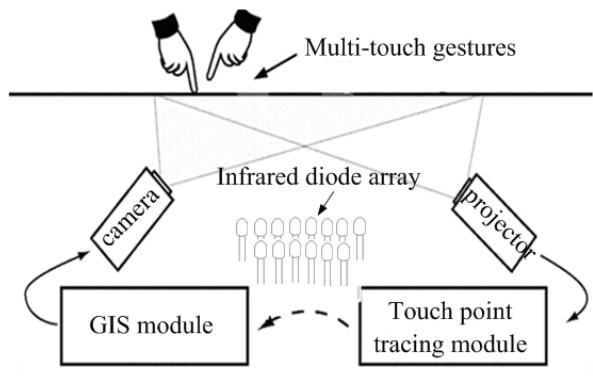

Fig. 1: Architecture of our multi-touch GIS.

\subsection{The workflow of multi-touch GIS}

Infrared light illuminates the touch plane from the bottom, and projector displays map on the touch screen. When fingers touch the surface, the light reflected at the touch point is stronger than the light reflected by the touch plane, so a shadow is occurred and captured by camera. These shadows are recognized as touch points by tracing module. Touch point data is converted into data packet which complies with TUIO protocol ${ }^{[10][11]}$ by touch point tracing module, and then is sent to the GIS module by socket communication. GIS module is responsible for receiving data packets, and analyzing data packet to obtain id, coordinate, speed, and acceleration of the touch point. GIS module ana- 
lyzes the shape of the trajectory according to the status of touch point and coordinates for recognizing gestures. Then GIS module converts the gesture to corresponding GIS operation, and completes operation by MapX, and finally displays the final results on touch screen through the projector.

\section{Touch point tracing algorithm}

Touch point tracing algorithm is used to trace the touch point and capture trajectory of touch point. The algorithm consists three parts: image processing, touch point contour extraction and touch point tracing. Firstly, the video image is processed by the following steps of removing background, image dynamic adjustments, smoothing, blur and so on. These operations can make the touch point in the image more distinct. Secondly, the outer contour of all touch points is extracted and the $\mathrm{X}$ and $\mathrm{Y}$ coordinate of each contour center is calculated and saved. Thirdly, the touch point tracing algorithm distinguishes which points are new and which points already existed according the move distance of touch point in the two consecutive images and identify these touch points finally. The process of touch point tracing algorithm is shown in Figure 2.

The part of image processing is implemented as the following steps:

- Removing the background image. The first frame of the video is saved as the background image. The rest frame of the image is subtracted by the background image to obtain grayscale image.

- Adjusting Image background dynamically. Since the video data from the camera and the ambient light is unstable, a linear transformation of the background image is made to correct its grayscale value, so that the background image and the current frame image can match each other in the gray feature, such as brightness and contrast.

- Binaryzation processing. The suitable threshold found by OSTU algorithm is used to carry out the gray value division processing of the image, which can make a stronger contrast of image.

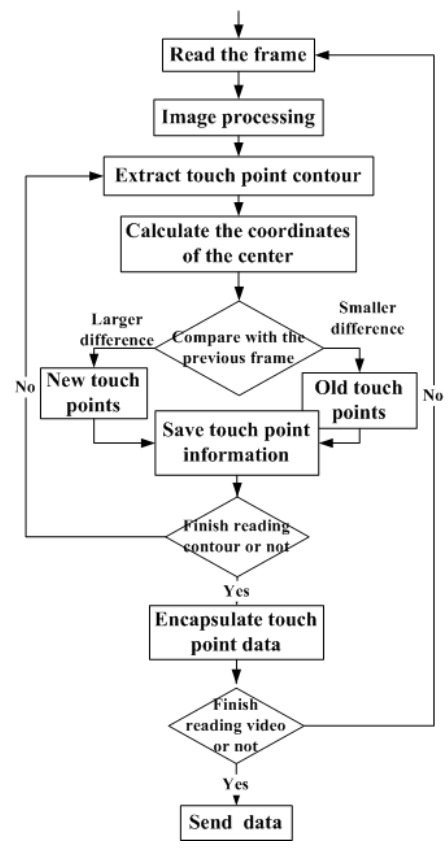

Fig. 2: The process of tracing algorithm.

- Smooth and high-pass blur processing. Firstly, a median filter is used to smooth image, which can remove the interference of background in the image. Then the sharpening filter is used for highpass obfuscation image, which can strengthen the outline of the image, remove the fuzzy part of the image, and leave a clear and bright image of touch points.

The part of touch point contour extraction is implemented as the following steps: 
- The extraction of the contour of touch point: The contour of the touch point consists of the critical points which have relatively large changes in the gray value in the image. Touch point contour extraction algorithm extracts the outer contour of all the touch points in the binary image based on contour edges threshold, and save the contour extracted.

- The exclusion of invalid points: At first, the contour area of all touch points is calculated; then the touch point whose area exceed the predefined threshold is treated as invalid touch point and should be removed.

- The calculation of the center of touch point: The center of each contour is calculated by the following zero-order moment formula, and then the coordinates of the contour center are obtained. In the equation (1), $\left(x_{0}, y_{0}\right)$ represents the coordinate of the contour center, $x$, $y$ represent coordinates of the pixel in the contour, $I(x, y)$ represents the pixel gray value.

$$
\left(x_{0}, y_{0}\right)=\left(\frac{\sum_{x} \sum_{y} x I(x, y)}{\sum_{x} \sum_{y} I(x, y)}, \frac{\sum_{x} \sum_{y} y I(x, y)}{\sum_{x} \sum_{y} I(x, y)}\right)
$$

The part of touch point tracing: As the sampling time of two adjacent frames is very short (for example, in the case of 30 frames per second, the sampling interval is about $33 \mathrm{~ms}$ ), and the touch point moving distance is limited during this period, the touch point tracing module determines which touch points are new and which points already existed according to the moving distance of the touch point in two adjacent frames, so it saves the touch point information to complete tracing the trajectory of the finger.

\section{Multi-touch gestures and state tran- sition model}

The multi-point touch interaction gestures can be divided into static gestures and dynamic gestures according to the finger movement state on the touch screen. Static gestures refer to still finger touch, and dynamic gesture refers to the movement of the finger corresponding to a trajectory in the touch screen. According to the number of touch points, the multi-point touch interaction gestures can be divided into single point gesture and multi-point gestures. According the two characteristics, the multi-touch gestures can be defined as single-point and multi-point gestures, touching and moving gestures ${ }^{[12]}$. At the same time, gestures needed to be mapped to the specific operation in GIS. Table 1 gives the gestures defined in our system and corresponding GIS operations.

Tab. 1: Multi-touch operation gesture.

\begin{tabular}{|c|c|c|c|}
\hline State & $\begin{array}{c}\text { Gestures } \\
\text { name }\end{array}$ & Finger movements & $\begin{array}{c}\text { Map manip- } \\
\text { ulations }\end{array}$ \\
\hline 0 & Empty & No & No touch \\
\hline 1 & $\begin{array}{l}\text { Single } \\
\text { finger } \\
\text { touch }\end{array}$ & $\begin{array}{l}\text { Static single finger } \\
\text { touch }\end{array}$ & Select object \\
\hline 2 & $\begin{array}{l}\text { Single } \\
\text { finger } \\
\text { panning }\end{array}$ & $\begin{array}{l}\text { Finger move along } \\
\text { the horizontal, } \\
\text { vertical or diagonal } \\
\text { direction }\end{array}$ & $\begin{array}{c}\text { Move map in } \\
\text { different } \\
\text { directions }\end{array}$ \\
\hline 3 & $\begin{array}{l}\text { Single } \\
\text { finger } \\
\text { polyline } \\
\text { moving }\end{array}$ & $\begin{array}{l}\text { Finger move along } \\
\text { the polyline }\end{array}$ & $\begin{array}{l}\text { Calculating } \\
\text { the distance } \\
\text { between some } \\
\text { points }\end{array}$ \\
\hline 4 & $\begin{array}{c}\text { Two-finger } \\
\text { touch }\end{array}$ & $\begin{array}{l}\text { Static two-finger } \\
\text { touch }\end{array}$ & $\begin{array}{l}\text { Calculate the } \\
\text { distance be- } \\
\text { tween two } \\
\text { points }\end{array}$ \\
\hline 5 & $\begin{array}{l}\text { Two fin- } \\
\text { gers sepa- } \\
\text { rated }\end{array}$ & $\begin{array}{l}\text { Two fingers depart } \\
\text { from each other at } \\
\text { the same time }\end{array}$ & $\begin{array}{c}\text { Map zoom } \\
\text { out }\end{array}$ \\
\hline 6 & $\begin{array}{l}\text { Two fin- } \\
\text { gers ap- } \\
\text { proach }\end{array}$ & $\begin{array}{l}\text { Two fingers move } \\
\text { toward each other } \\
\text { at the same time }\end{array}$ & Map zoom in \\
\hline
\end{tabular}

According to Table 1, we can establish a state transition model shown in Figure 3. When the system accepts touch events, it can be transferred from one state to another. The initial state is empty state expressed as the state 0 . When one finger 
touches screen, the system can enter in a single touch state, the state 1 , which can simulate a mouse click operation. Then it can jump into the state 2 or 3 in accordance with the shape of the finger movement on the screen, and returns to state 1 if the touch point stops moving. In the case of state 1 , when another hand touches the screen, the system may enter in a state of two-finger touch, the state 4 . Then it can move from the state 4 to the state 5 or 6 according to the two fingers respectively moving direction, and returns to state 4 if the touch points stop moving. When there is one finger away from the screen, the system will be transferred in accordance with the state of the remaining finger, and when all fingers away from the screen, it returns to state 0 .

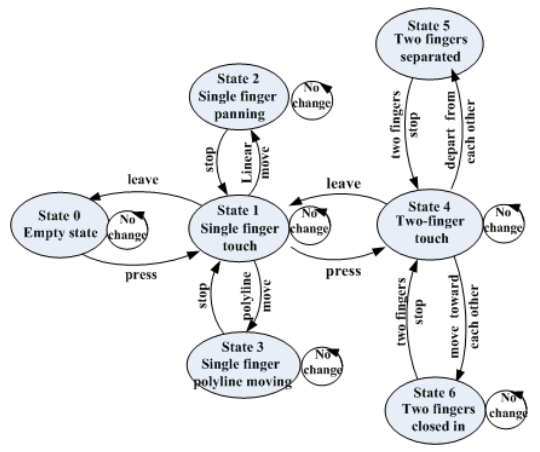

Fig. 3: The state transition model of gesture.

\section{Gesture recognition method based on touch point trajectory}

A gesture recognition method based on touch point trajectory is given according to the number of the touch points and state of the touch gesture. The method collects and processes the coordinates of the touch points for real-time. After gesture operation is completed (all touch points away from the touch screen), the touch gestures result is output on the touch screen. Response time is relatively short, and the correct identification rate is high where the gesture is static gestures and dynamic gestures. Gesture recognition method flowchart shows in Figure 4.

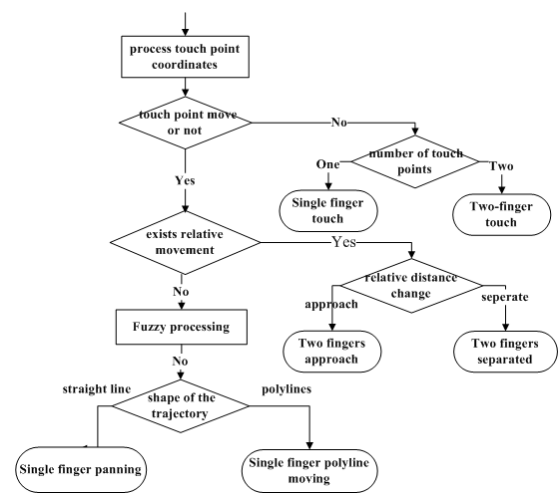

Fig. 4: Gesture recognition method flowchart.

In the recognition algorithm, a touch point is treated as valid touch point if its residence time is within a time range 800$1200 \mathrm{~ms}$. For effective touch point, according to whether the moving distance of touch point within the range of the threshold value s1 to determine the gesture is dynamic gestures or static gestures. If the moving distance of touch point is less than s1, the gesture is static gesture, otherwise dynamic gesture.

For static gestures, we determine gestures based on the number of touch points. If the number of touch points is 1 , it is a single finger touch gesture. If the number of touch points is 2 , it is a twofinger touch gesture. Otherwise, it is an invalid gesture. For dynamic gesture, we determine whether relative movement exists based on whether the relative movement distance between the touch points is greater than the threshold value s2.

If relative movement does exist, according to the change of distance between the touch points, the gesture is recognized as two fingers separation gestures or two fingers approach gesture. If the relative distance becomes larger, it 
is the separation gestures; otherwise it is approach gesture. If no relative movement exists, the trajectory of touch points is fuzzed as a trajectory of single touch point, and then gesture is judged based on the shape of the effective trajectory. If the trajectory is a polyline, it means single finger polyline gesture. If the trajectory is a straight line, the gesture is a single finger pan gesture. For the single finger pan gesture, the direction of movement can be determined by comparing the slope of the line, $\mathrm{K}$, with the threshold $\mathrm{Kx}$ and $\mathrm{Ky}$, as shown in Figure 5. Table 2 shows the different slope of touch point trajectory corresponding to different panning gesture.

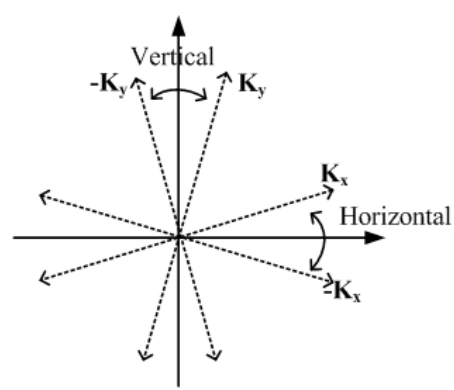

Fig. 5: The slope threshold range.

Tab. 2: Different slope of touch point trajectory corresponding to different panning gesture.

\begin{tabular}{ll}
\hline $\begin{array}{l}\text { Threshold value } \\
\text { range }\end{array}$ & Touch points panning gesture \\
\hline$-\mathrm{Kx}<\mathrm{K}<\mathrm{Kx}$ & Horizontally move gestures \\
$\mathrm{K}<-\mathrm{Ky}, \mathrm{K}>\mathrm{Ky}$ & $\begin{array}{l}\text { Vertically move gestures } \\
\mathrm{Kx}<\mathrm{K}<-\mathrm{Ky}\end{array}$ \\
$\begin{array}{l}\text { The top-right gestures or the } \\
\text { bottom-left gestures }\end{array}$ \\
$-\mathrm{Ky}<\mathrm{K}<-\mathrm{Kx}$ & $\begin{array}{l}\text { The bottom-right gestures or } \\
\text { the top-left gestures }\end{array}$ \\
\hline
\end{tabular}

The gestures defined in table 1 are tested 500 times in recognition experiment on our multi-touch GIS platform. The correct rate of recognition algorithm with different thresholds is shown in Table 3 and Table 4.
Tab. 3: The recognition experimental results 1 .

\begin{tabular}{llll}
\hline \multicolumn{1}{c}{ Threshold } & $\begin{array}{l}\text { Single } \\
\text { finger } \\
\text { touch }\end{array}$ & $\begin{array}{l}\text { Single } \\
\text { finger } \\
\text { Panning }\end{array}$ & $\begin{array}{l}\text { Single } \\
\text { finger } \\
\text { polyline } \\
\text { moving }\end{array}$ \\
\hline $\begin{array}{l}\mathrm{K} x=0.01, \mathrm{Ky}=1 \\
\mathrm{~s} 1=1, \mathrm{~s} 2=2\end{array}$ & $52 \%$ & $35 \%$ & $25 \%$ \\
$\begin{array}{l}\mathrm{K} x=0.10, \mathrm{Ky}=4 \\
\mathrm{~s} 1=100, \mathrm{~s} 2=40\end{array}$ & $80 \%$ & $85 \%$ & $82 \%$ \\
$\begin{array}{l}\mathrm{K} x=0.2, \mathrm{Ky}=6 \\
\mathrm{~s} 1=300, \mathrm{~s} 2=100\end{array}$ & $99 \%$ & $96 \%$ & $95 \%$ \\
$\begin{array}{l}\mathrm{Kx}=0.5, \mathrm{Ky}=10 \\
\mathrm{~s} 1=400, \mathrm{~s} 2=150\end{array}$ & $99 \%$ & $90 \%$ & $94 \%$ \\
$\begin{array}{l}\mathrm{K} x=1, \mathrm{Ky}=15 \\
\mathrm{~s} 1=500, \mathrm{~s} 2=200\end{array}$ & $99 \%$ & $80 \%$ & $85 \%$ \\
\hline
\end{tabular}

Tab. 4: The recognition experimental results 2 .

\begin{tabular}{|c|c|c|c|}
\hline Threshold & $\begin{array}{l}\text { Two- } \\
\text { finger } \\
\text { touch }\end{array}$ & $\begin{array}{l}\text { Two fin- } \\
\text { gers sep- } \\
\text { arated }\end{array}$ & $\begin{array}{l}\text { Two fin- } \\
\text { gers ap- } \\
\text { proached }\end{array}$ \\
\hline $\begin{array}{l}\mathrm{Kx}=0.01, \mathrm{Ky}=1 \\
\mathrm{~s} 1=1, \mathrm{~s} 2=2\end{array}$ & $50 \%$ & $48 \%$ & $46 \%$ \\
\hline $\begin{array}{l}\mathrm{Kx}=0.10, \mathrm{Ky}=4 \\
\mathrm{~s} 1=100, \mathrm{~s} 2=40\end{array}$ & $70 \%$ & $95 \%$ & $92 \%$ \\
\hline $\begin{array}{l}\mathrm{Kx}=0.2, \mathrm{Ky}=6 \\
\mathrm{~s} 1=300 \mathrm{~s} 2=100\end{array}$ & $97 \%$ & $98 \%$ & $98 \%$ \\
\hline $\begin{array}{l}\mathrm{Kx}=0.5, \mathrm{Ky}=10 \\
\mathrm{~s} 1=400 \mathrm{~s} 2=150\end{array}$ & $96 \%$ & $97 \%$ & $96 \%$ \\
\hline $\begin{array}{l}\mathrm{Kx}=1, \mathrm{Ky}=15 \\
\mathrm{~s} 1=500 \mathrm{~s} 2=200\end{array}$ & $96 \%$ & $82 \%$ & $80 \%$ \\
\hline
\end{tabular}

According to the data in Table 3 and Table 4 , we can make a conclusion that the correct rate of gesture recognition depends on the threshold $s 1, s 2, K x$ and $K y$. The user can adjust these thresholds according to the requirements of the client application to make the correct rate match the requirement. In the case of $K x=0.2, \quad K y=6, \quad s l=300, \quad s 2=100, \quad$ the gesture recognition algorithm in this article has achieved relatively high recognition accuracy.

In the process of gesture recognition, we need to record the corresponding touch point coordinates which are used to provide accurate position data for the click operation and calculate the distance between two points. If the gesture is a single finger polyline moving gestures, we also need to record the coordinates of the knee points. We don't need record the coordinates for the other gestures. 


\section{Experiment Result}

The user can operate our multi-touch GIS directly by using fingers to touch and move on the touch plane. Figure 6 shows the actual running effect of the multitouch GIS system. We select two typical touch operations to exhibit our experiment result. In Figure 6 the blue lines represent the trajectory of touch point and the white circle at the end of trajectory represent the moving touch point. As shown in Figure 6 (a), the map can be zoomed in through two fingers simultaneously departing from each other. As shown in Figure 6 (b), the map can be dragged by one finger moving linearly along the horizontal, vertical or inclined direction.

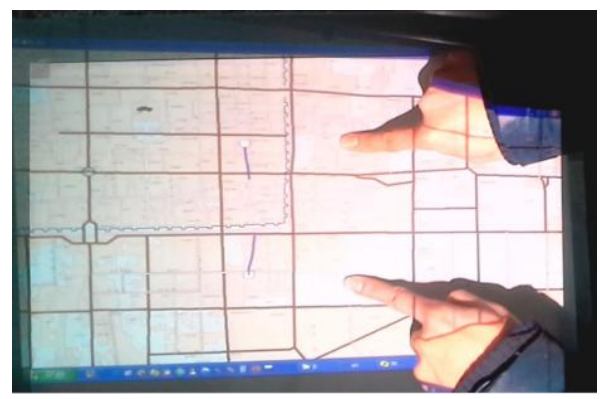

(a). Zoom in map by two fingers separated gesture.

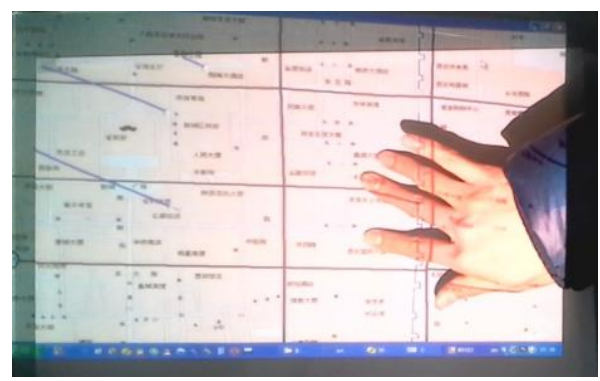

(b). Drag map by moving fingers linearly.

Fig. 6: The actual running effect of the multitouch GIS system.

\section{Conclusion and future works}

This paper describes a GIS based on multi-touch technology. The system uses the Rear-DI technology to build multi-touch platform, and transfer touch point data by TUIO protocol between the tracing module and GIS module. A series of image processing and computing of video frames is used to get data and trajectory of touch point according to the characteristics of touch point. Some multi-touch gestures corresponding to typical GIS operation and its state transition model are defined in the GIS module. According to these definitions, a gesture recognition method based on geometric characteristics of trajectory is given. This method can recognize the trajectory into correct interaction gestures and operate the GIS through MapX. In addition, due to the multi-touch human-computer interaction technique, the system has better manmachine interactive feature and user experience relative to the traditional GIS system.

There are possible future extensions of this work. For the convenience of experiment, the touch screen of our GIS is smaller, so the stability and correct rate need to be tested further in large touch screen case. In addition, there are only six gestures defined in our GIS, which are not enough for a complete GIS. So, more operating gestures need to be defined to improve the function of the system.

\section{Acknowledgements}

This thesis was joint supported by the Shaanxi Provincial Natural Science Foundation project (Grant No.2011JQ801 5), Xidian University Fundamental Research (Grant No.72005581, 72114346) and the Xi'an Science and Technology research project (Grant No.CXY1118 (2)). 


\section{References}

[1] Alex Teiche, Ashish Kumar Rai, Chris Yanc, etc, "Chinese Version of Multi-touch Technologies Handbook," Haiyun Zhang, Dezhi Liu, Zhenbang Zhang annotation, www.mt21.com, 2009.

[2] Jefferson H, "Low-cost multi-touch sensing through frustrated total internal reflection," Proc. Of the 18th Annual ACM Symposium on User Interface Software and Technology, pp. 315-319, 2005.

[3] Yue Shi, Chun Yu, Yuanchun Shi, "Finger Gesture Interaction on Large Tabletop for Sharing Digital Documents among Multiple Users," Proc. Of The 2008 First IEEE International Conference on Ubi-media Computing, pp. 8-13, 2008.

[4] Huxiong Liao, Songyang Lao, Hongtao Shao, Gang Liu, "Application Research on Touch-based Twohanded Natural Interaction Command Technology," JOURNAL OF NATIONAL UNIVERSITY OF DEFENSE TECHNOLOGY, pp. 117-122, 2011.

[5] Sangbong, Park, "Implementation of new gestures on the Multi-touch table," Proc. Of The 2012 International Conference on ICT Convergence (ICTC), pp. 168-169, 2012.

[6] Youngseok Ahn, Jun Lee, Woohyon Kim, Jee-In Kim, Wook Chang,
HyungSeok Kim, Jihyung Park, “A slim hybrid multi-touch tabletop interface with a high definition LED display and multiple cameras," Proc. Of The 2012 IEEE International Conference on Consumer Electronics (ICCE), pp. 217-218, 2012.

[7] Chengzhi Xu, Chengyu Xu, Tieyun Qian, "New Spatial Query Language Based on GIS," Computer Science, pp. 206-210, 2010.

[8] Dewen Wang, Wenqing Zhao, "Design and Implementation of Geographic Information System Based on MapX," Microcomputer Development, pp. 59-61, 2003.

[9] Xueyuan Song, Jianwen Li, Guoyuan Ma, Zhulong Sun, Junzheng Li, “GPS Position Information Real-time Mark System Based on MapX," HYDROGRAPHIC SURVEYING AND CHARTING, pp. 58-60, 2011.

[10] http://tuio.org/.

[11] Kaltenbrunner M, Bovermann T, Bencina R, Costanza E, "TUIO - A Protocol for Table-Top Tangible User Interfaces," Proc. Of the 6th International Workshop on Gesture in $\mathrm{Hu}$ man-Computer Interaction and Simulation (GW 2005), 2005.

[12] Yunxiang Ling, Guohua Zhang, Rui Li, Ting Ye, "Research on Natural Gesture Recognition Method Based on Multi-touch," JOURNAL OF NATIONAL UNIVERSITY OF DEFENSE TECHNOLOGY, pp. 127-132, 2010. 